\title{
Anomalous Radiative Trapping in Laser Fields of Extreme Intensity
}

\author{
A. Gonoskov, ${ }^{1,2,3,{ }^{*}}$ A. Bashinov, ${ }^{2,3}$ I. Gonoskov, ${ }^{4}$ C. Harvey, ${ }^{5}$ A. Ilderton, ${ }^{1}$ A. Kim, ${ }^{2,3}$ \\ M. Marklund, ${ }^{4,1}$ G. Mourou, ${ }^{3,6}$ and A. Sergeev ${ }^{2,3}$ \\ ${ }^{1}$ Department of Applied Physics, Chalmers University of Technology, SE-41296 Gothenburg, Sweden \\ ${ }^{2}$ Institute of Applied Physics, Russian Academy of Sciences, Nizhny Novgorod 603950, Russia \\ ${ }^{3}$ University of Nizhny Novgorod, Nizhny Novgorod 603950, Russia \\ ${ }^{4}$ Department of Physics, Umeå University, SE-90187 Umeå, Sweden \\ ${ }^{5}$ Centre for Plasma Physics, Queen's University Belfast, Belfast BT7 1NN, United Kingdom \\ ${ }^{6}$ Institut de la Lumière Extrême, UMS 3205 ENSTA, Ecole Polytechnique, CNRS, 91761 Palaiseau, France
}

(Received 5 August 2013; published 2 July 2014)

\begin{abstract}
We demonstrate that charged particles in a sufficiently intense standing wave are compressed toward, and oscillate synchronously at, the antinodes of the electric field. We call this unusual behavior anomalous radiative trapping (ART). We show using dipole pulses, which offer a path to increased laser intensity, that ART opens up new possibilities for the generation of radiation and particle beams, both of which are high energy, directed, and collimated. ART also provides a mechanism for particle control in high-intensity quantum-electrodynamics experiments.
\end{abstract}

DOI: 10.1103/PhysRevLett.113.014801

PACS numbers: 41.60.-m, 52.38.-r, 52.59.-f

Introduction.-Progress in laser technology has opened up possibilities for creating ultraintense light sources [1-3] with the aim of studying phenomena at the interface of high-field and high-energy physics [4]. Among these, QED effects and radiation dominated particle dynamics are of great interest and are guiding the direction of upcoming laser programs [5-7].

In this Letter we report the existence of a new regime of charged particle dynamics in ultraintense light. We show that particles in a sufficiently intense standing wave are compressed toward, and oscillate synchronously at, the maxima of the electric field rather than the minima. This unusual behavior, which we call anomalous radiative trapping (ART), is due to radiation friction. We demonstrate in a specific geometry that ART can be used for particle control $[8,9]$ for studying fundamental physics [10,11], and for the generation of multi-GeV, directed, gamma rays [12] and collimated, energetic particle beams [13].

Anomalous radiative trapping.-We begin by simulating the relativistic dynamics of (preexisting, initially uniformly distributed) particles in electromagnetic plane standing waves of various amplitudes, and calculate the particles' long-term spatial distribution. Particle motion is due to both the Lorentz force and the particle's own recoil when it emits radiation, an effect which rises with intensity. Motion is planar, as both forces act only in the plane transverse to the magnetic field. Our code contains a relativistic particle pusher, propagating electrons according to the Lorentz equation. Emission and recoil are implemented at each time step according to quantum theory via statistical routines, using inverse sampling [14,15]. See Ref. [14] for a description of the event generator and Ref. [16] for the probability of emission in ultraintense fields. In Fig. 1(a) we plot the long-term particle distribution as a function of the wave amplitude. The distribution was extracted at the instant of vanishing electric field after 100 oscillations of the standing wave, when it was observed that the distribution had stabilized. From here on, we write "node" (antinode) to mean "electric field node" (antinode), and measure position $x$, time $t$, and field strength $a$ in units of $\lambda / 2 \pi, \lambda / 2 \pi c$, and $2 \pi m c^{2} / \lambda e$, respectively, where $\lambda$ is the standing wave wavelength.

The simulations show various trapping phenomena. At low intensities, we see trapping in the minima of the ponderomotive potential (describing the average effect of the Lorentz force), coinciding with the positions of the nodes. Because of relativistic effects, electrons are released from this ponderomotive trapping and move chaotically (except in relativistic reversal $[17,18]$ ) as the field amplitude rises $[19,20]$. As the role of radiation losses increases, it is known that, following "phase space contraction" [21], the particles subsequently become trapped once more, and again in the electric field nodes $[19,22]$. We call this effect normal radiative trapping (NRT). But remarkably, at even higher intensities, the electrons become focused toward and trapped around the positions of the antinodes, i.e., at the maxima of the ponderomotive potential. We call this counterintuitive behavior anomalous radiative trapping.

To understand ART, we first assess the relevance of quantum effects. Compare the distribution of Fig. 1(a) with that in Fig. 1(b), which was obtained from an entirely classical simulation using the Landau-Lifshitz equation to describe a radiating particle; it is clear that while the quantum nature of emission causes a broadening of the particle distributions, the trapping phenomena are present both with and without quantum effects. (We can conclude, 


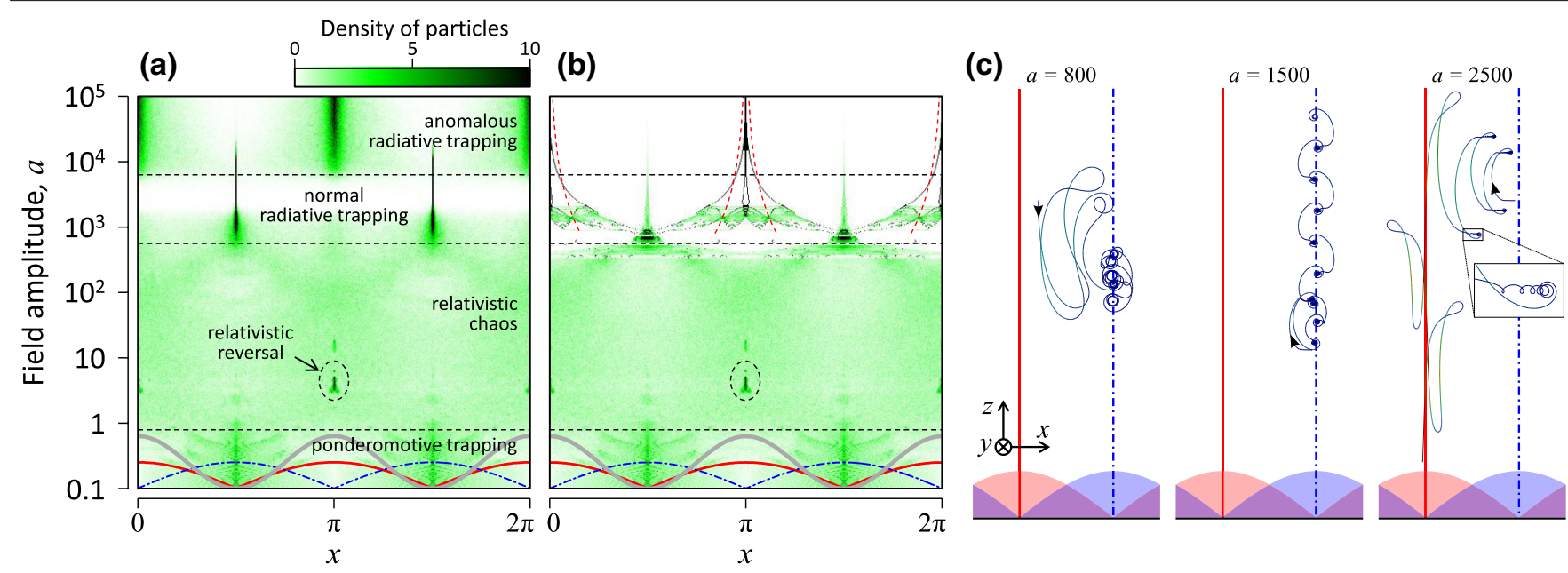

FIG. 1 (color online). (a) The long-term density distribution of electrons in a standing wave as a function of wave amplitude $a$. The spatial distribution of the electric and magnetic fields, and the ponderomotive potential, are sketched at the bottom with red (solid thin), blue (dot-and-dash), and gray (solid thick) curves, respectively. $\mathbf{E}$ (B) is orientated along the $z$ axis ( $y$ axis). Radiation reaction is included via quantum emission. (b) The same density distribution calculated using classical radiation reaction. (c) Typical particle trajectories in the $x-z$ plane. The first and third are in the NRT and ART regimes, respectively, while the second shows the transition between them. Solid red (dot-and-dash blue) lines show the locations of antinodes (nodes).

because the stochastic nature of quantum radiation loss does not spoil ART, that the effect is not sensitive to the particles' initial position or momentum.) We therefore proceed to explain the observed trapping phenomena in terms of classical radiation reaction.

In the ultrarelativistic limit, radiation losses are determined predominantly by a particle's acceleration transverse to its velocity [23]. The magnetic component of the Lorentz force is always transverse to velocity, whereas the electric component accelerates parallel to $\mathbf{E}$, meaning its contribution to transverse acceleration depends on the relative orientation of the field and particle velocity. On average, then, the rate of radiative loss is higher in the vicinity of the nodes. In the NRT regime, this causes particles to lose their energy and rotate close to the nodes, as illustrated by the first trajectory in Fig. 1(c). This trajectory also shows the irregularity of motion in the NRT regime.

As the wave amplitude rises, the relative role of radiation losses increases and the particles can lose essentially all of their energy within just a fraction of the wave period. As a result, motion becomes more regular, see the second trajectory in Fig. 1(c), containing alternating phases of acceleration (starting from almost zero energy) whenever the electric field peaks, and deceleration (almost to rest) whenever the magnetic field peaks. The regularity of this "radiation dominated motion" $[24,25]$ is key to the particles' net migration toward the antinodes, as shown in the third trajectory of Fig. 1(c), by the following mechanism.

In the ART regime, particles follow, in part, paths described by the green curves in Fig. 2, gyrating with drift velocity $c \mathbf{E} \times \mathbf{B} / B^{2}$ when $B>E$, or moving essentially linearly with velocity going to $c \mathbf{E} \times \mathbf{B} / E^{2}$ when $E>B$. These two types of motion would describe particle motion in the low-energy limit; particles would be shifted toward the antinode as the electric field was rising, and away from it as the electric field was falling. As these stages (call them stage I and stage II) are symmetric, particles would oscillate in the low-energy limit, but would not migrate to any certain position. In the ART regime, though, radiative losses lead to an asymmetry in these two stages. The particle gains energy due to acceleration by the electric field and, as explained above, loses it mostly due to the magnetic field; thus, the gamma factor is larger in stage II than in stage I. A higher gamma factor during stage II means that the particle resists the magnetic field, so the shift away from the antinode is smaller in stage II than the shift toward the

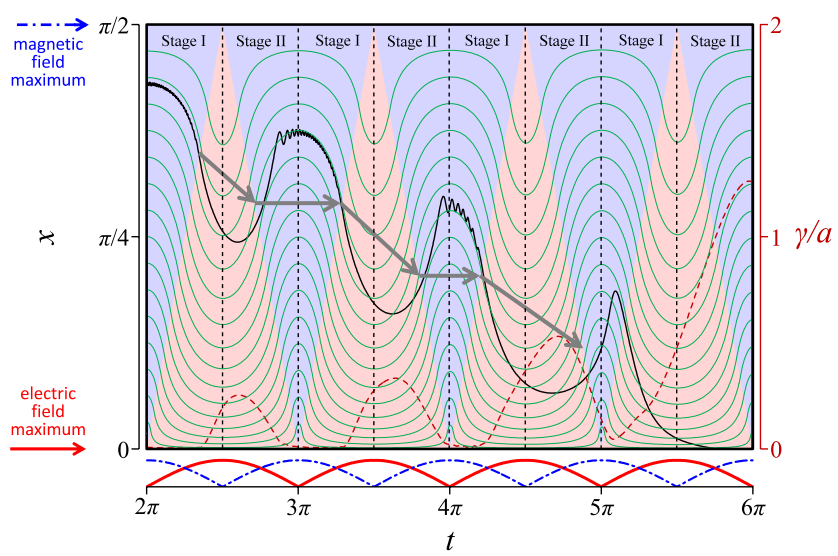

FIG. 2 (color online). Typical particle trajectory (black solid curve) and gamma factor (red dashed curve) in the ART regime. Red (blue) regions correspond to electric (magnetic) field dominance, $E>B(B>E)$. Thin green lines describe the low-energy limit. 
antinode in stage I. As a result, particles in the ART regime migrate toward the antinodes within a few oscillations of the standing wave. The final trajectory of Fig. 1(c) shows migration in around three cycles.

When particles reach the vicinity of the antinodes they sit on stable attractors. The detailed form of the trajectories is extremely sensitive to initial conditions and intensity. However, we can give a broad analysis of the ultrarelativistic motion and in doing so estimate the spatial spread of trapped particles. Let the standing wave have field components $E_{z}=a \cos x \cos t$ and $B_{y}=-a \sin x \sin t$. We estimate the spread $x_{r}$ as the distance the magnetic field can drag particles away from the antinodes. Beginning with a particle at $x_{r} \simeq 0$ (near an antinode) at $t=0$, the magnetic field strength is $B_{y} \approx a x_{r} \sin t$, and this will be larger than the electric field at the same point, $E \approx a \cos t$, for a short time $\approx 2 x_{r}$ around $t=\pi / 2$. As the job of the magnetic field here is to rotate the particle back toward the antinode, we assume that the particle describes a half circle in time $2 x_{r}$. When the magnetic field dominates, we also assume that motion is effectively "rotation in a magnetic field," with synchrotron frequency $\omega_{s}=a x_{r} / \gamma_{r}$, (this is dimensionless $e B / m c \gamma$ ) for typical gamma factor $\gamma_{r}$. Equating the halfperiod $\pi / \omega_{s}$ with $2 x_{r}$ yields $\gamma_{r}=2 a x_{r}^{2} / \pi$. Finally, we assume that the Lorentz force $\left(\approx a x_{r}\right)$ field is roughly balanced by the dominant term in the radiation reaction force,

$$
a x_{r} \approx \frac{4 \pi}{3} \frac{r_{e}}{\lambda} \gamma_{r}^{2} a^{2} x_{r}^{2}
$$

Eliminating $\gamma_{r}$ gives

$$
x_{r} \approx 0.9\left(\frac{\lambda}{a^{3} r_{e}}\right)^{1 / 5}
$$

This rough estimate for the particle spread, shown at the top of Fig. 1(b) with dashed red lines, clearly fits the numerical results well and explains why particles are concentrated toward the antinode with rising amplitude.

The classical equations of motion have, in the ultrarelativistic regime, a similarity parameter $\delta=\left(r_{e} / \lambda\right) a^{3}$ [24] defining the transition between relativistic stochastic motion and the regimes of NRT and ART. Based on the data of Fig. 1(b), we can identify the threshold values of $\delta$ for both regimes, $\delta_{t h}^{\mathrm{NRT}} \approx 0.5, \delta_{t h}^{\mathrm{ART}} \approx 600$, corresponding to threshold intensities in terms of $I=(c / 8 \pi) E_{\max }^{2}$ :

$$
\begin{aligned}
& I_{\mathrm{th}}^{\mathrm{NRT}} \approx 5 \times 10^{23} \frac{W}{\mathrm{~cm}^{2}}\left(\frac{0.81 \mu \mathrm{m}}{\lambda}\right)^{4 / 3}, \\
& I_{\mathrm{th}}^{\mathrm{ART}} \approx 6 \times 10^{25} \frac{W}{\mathrm{~cm}^{2}}\left(\frac{0.81 \mu \mathrm{m}}{\lambda}\right)^{4 / 3} .
\end{aligned}
$$

[While Eq. (2) gives $\delta>1$ for trapping near the antinode, that estimate is based on assumptions that are valid only in the ART regime, at $\delta>600$.]
Particle motion in dipole waves.-High field strengths are thus needed to observe NRT and ART, but future facilities will likely use multiple colliding pulses [3] to produce intense fields which do not have a simple standing wave structure. Does ART exist in such beams?

Given fixed input power, the electric field strength in a laser focus can be maximized by using a dipole pulse [26], which saturates the upper bound on focusing efficiency [27]. It describes a converging wave of light, which can be pictured as the time-reversed process of emission from a dipole antenna. Using several identical channels to mimic a dipole pulse is the optimal design for future facilities and offers the potential for going beyond current field strength and intensity records $[28,29]$. Figure 3 shows a focusing concept based on 12 colliding pulses, and which gives less than just 10\% deviation from the exact distribution of electric field strength (see the Supplemental Material [30]). We will show that such deviations do not prevent NRT and ART from appearing; thus, we will proceed by simulating an exact dipole pulse corresponding to that generated by laser pulses with a Gaussian profile of 30 fs duration (FWHM for intensity), wavelength $\lambda=810 \mathrm{~nm}$, and peak total power of $200 \mathrm{PW}$ (averaged over the central period), as is expected to be available at future international projects $[2,3]$.

We again begin the simulation with a uniform distribution of electrons. The mutual Coulomb interaction is neglected, but will be addressed below. In Fig. 4(a) we plot the time evolution (top to bottom) of the electron density in

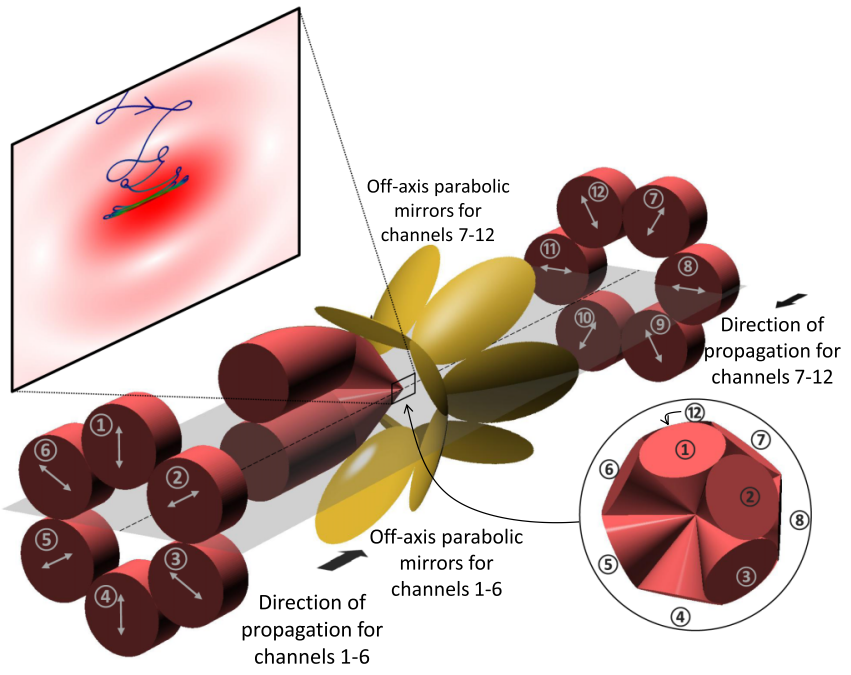

FIG. 3 (color online). Focusing concept for dipole wave production and the ART effect. Two sets of six counterpropagating beams (polarization shown with double-ended arrows) are reflected by two sets of parabolic mirrors (yellow) aligned such that their surfaces lie along a paraboloid. Both paraboloids have the same symmetry axis (dashed line, $z$ axis) and the same focus point. The resulting focal beam structure is shown in the inset. Top left: The focal electric field (schematically) and a typical electron trajectory in the ART regime. The particle becomes trapped around the peak of the electric field. 


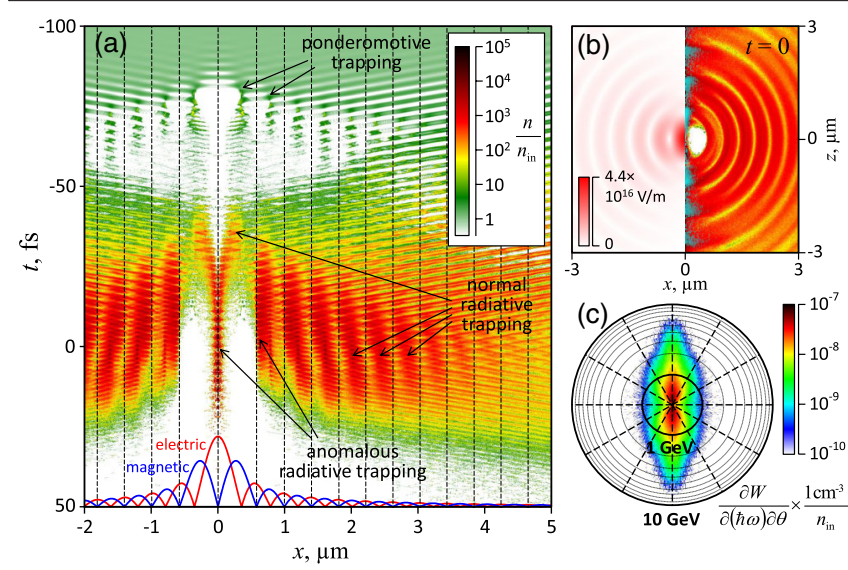

FIG. 4 (color online). Simulation results for electron motion in the dipole wave. (a) Time evolution of the electron density (divided by the initial density) on the $x$ axis, $z=0$. Peak electric field locations are shown with dashed lines. (b) Electric field strength distribution in the dipole wave (left-hand side) and particle density distribution (right-hand side) at the instance of peak field strength; photons with energy exceeding $3 \mathrm{GeV}$ are shown in cyan. (c) Photon emission distribution as a function of angle and energy (radial coordinate, log scale).

the focus, $z=0$, as a function of transverse position $x$. The particles' motion is confined to the plane passing through the axis of rotational symmetry ( $z$ axis), since both the Lorentz force and recoil are orientated in this plane. When the front edge of the pulse reaches the center, it first forms a standing wave with moderate amplitude, and as the amplitude increases we see the accumulation and trapping of electrons in different regimes, as in the previous standing wave simulation. It is crucial to note that NRT and ART appear here at the same intensity thresholds as for the standing wave. This shows that the detailed structure of the field is not necessary for ART to appear; provided there is some standing-wave-like structure in the focus, the appearance of ART is dependent only on the attainable intensity.

We end this section by outlining some potential applications of ART. In the considered setup the particles are delivered to the region of trapping by the ponderomotive force at the front edge of the converging dipole pulse. Thus, the number of particles trapped in the vicinity of a particular antinode, which we refer to as being in a particular "trapping state," can be controlled by the shape of the pulse's front edge. The spatial structure of the pulse is such that the only channel for electrons to leave the trapping states is along, and in the vicinity of, the $z$ axis. (This is not related to the symmetry of the pulse, but rather to the surpassing of the above field strength thresholds.) As well as this wellcollimated source of highly energetic electrons, ART also provides a novel source of well-collimated hard photons. In Fig. 4(c) we plot the emitted photon distribution, with energies extending up to $6 \mathrm{GeV}$ (the maximum electron energy). One can distinguish the most energetic peak with energies above $3 \mathrm{GeV}$ and an angular spread of about $10^{\circ}$.
These photons, also shown in Fig. 4(b), are emitted by electrons in the central trapping state; see the video in the Supplemental Material [30]. Our simulations show that each electron emits on average 10 photons per cycle, of which one has an energy above $3 \mathrm{GeV}$. Triggering such sources of energetic particles and photons when surpassing the threshold intensities [Eq. (3)] can be used for the experimental verification of ART and NRT. Our simulations show that NRT can also lead to the formation of collimated electron and photon sources, but that these are less energetic than those in the ART regime.

Discussion.-Here, we address the impact of thus-far neglected effects, beginning with the Coulomb force. The central trapping state occupies a volume of radius $0.1 \mu \mathrm{m}$. The number of particles $N$ inside this volume is limited by their mutual Coulomb interaction. We therefore ask, how high can $N$ be before the Coulomb repulsion prevents another particle from entering the trapping state? To answer this, we equate the magnitude of the Lorentz force $\left(F_{L} \sim e E r\right)$ with that of the Coulomb force at the edge of the trapping state, $F_{C} \sim e^{2} N /\left(4 \pi r^{2}\right)$ with $r=0.1 \mu \mathrm{m}$. For the considered case, $(c / 8 \pi) E_{\max }^{2} \approx 2 \times 10^{26} \mathrm{~W} / \mathrm{cm}^{2}$ in the center, this yields $N \sim 10^{10}$. Data from Fig. 4 then let us estimate the maximum number of photons in the $1 \mathrm{GeV}$ range emitted by these electrons as $N_{h} \sim 10^{11}$, corresponding to a total energy ( $10 \mathrm{~J}$ ) of order $0.1 \%$ of the initial laser energy. These estimates illustrate the potential capabilities of the proposed setup; taking into account the mutual interaction of particular targets (solid drop, gas jet, etc.) can identify the real limitations and possibilities.

Conclusions. - In summary, we have shown that in focused fields (standing waves) of sufficiently high intensity, radiation damping causes particles to become trapped in, rather than expelled from, the antinodes of the electric field. This opens up new possibilities for hard photon generation, charged particle acceleration, and for studying QED.

Experimental demonstration of NRT is possible using a configuration of two counterpropagating pulses [22] or with optimal focusing; this would require a total power of 1-2 PW, which is within the reach of several current and proposed facilities [1]. ART could be demonstrated at proposed international high-intensity facilities such as ELI and XCELS, for which the dipole setup provides $I_{\max } \approx 2 \times 10^{26} \mathrm{~W} / \mathrm{cm}^{2}$, assuming $200 \mathrm{PW}$ total power.

The simulations were performed on resources provided by the Swedish National Infrastructure for Computing (SNIC) at HPC2N. The authors are supported by the Ministry of Education and Science of the Russian Federation, Agreement No. 11.G34.31.0011 (A. G., G. M., A.S.), the Russian Foundation for Basic Research Grant No. 12-02-12086 (A. G.), the Swedish Research Council, Contracts No. 2011-4221 (A. I.), No. 2010-3727 and No. 2012-5644 (M.M.), the European Research Council Contract No. 204059-QPQV (A. I., M. M.), and EPSRC Grant No. EP/I029206/1-YOTTA (C. H.). 
*arkady.gonoskov@chalmers.se

[1] Vulcan 10 PW, http://www.clf.stfc.ac.uk/.

[2] ELI, http://www.extreme-light-infrastructure.eu/.

[3] XCELS, http://www.xcels.iapras.ru.

[4] E. Esarey, C. B. Schroeder, W. P. Leemans, Rev. Mod. Phys. 81, 1229 (2009).

[5] T. Heinzl, B. Liesfeld, K.-U. Amthor, H. Schwoerer, R. Sauerbrey, and A. Wipf, Opt. Commun. 267, 318 (2006).

[6] G. Zavattini, U. Gastaldi, R. Pengo, G. Ruoso, F. Della Valle, and E. Milotti, Int. J. Mod. Phys. A 27, 1260017 (2012).

[7] HIBEF, http://www.hzdr.de/.

[8] C. Harvey and M. Marklund, Phys. Rev. A 85, 013412 (2012).

[9] M. Tamburini, C. H. Keitel, and A. Di Piazza, Phys. Rev. E 89, 021201(R) (2014).

[10] J. Jaeckel and A. Ringwald, Annu. Rev. Nucl. Part. Sci. 60, 405 (2010).

[11] A. Di Piazza, C. Müller, K. Z. Hatsagortsyan, and C. H. Keitel, Rev. Mod. Phys. 84, 1177 (2012).

[12] V. Malka, J. Faure, Y. A. Gauduel, E. Lefebvre, A. Rousse, and K. Ta Phuoc, Nat. Phys. 4, 447 (2008).

[13] S. Corde, K. Ta Phuoc, G. Lambert, R. Fitour, V. Malka, and A. Rousse, Rev. Mod. Phys. 85, 1 (2013).

[14] N. V. Elkina, A. M. Fedotov, I. Y. Kostyukov, M. V. Legkov, N. B. Narozhny, E. N. Nerush, and H. Ruhl, Phys. Rev. ST Accel. Beams 14, 054401 (2011).

[15] A. R. Bell and J. G. Kirk, Phys. Rev. Lett. 101, 200403 (2008).

[16] V. I. Ritus, J. Russ. Laser Res. 6, 497 (1985).

[17] A. E. Kaplan and A. L. Pokrovsky, Phys. Rev. Lett. 95, 053601 (2005).

[18] I. Y. Dodin and N. J. Fisch, Phys. Rev. E 77, 036402 (2008).
[19] G. Lehmann and K. H. Spatschek, Phys. Rev. E 85, 056412 (2012).

[20] D. Bauer, P. Mulser, and W.-H. Steeb, Phys. Rev. Lett. 75, 4622 (1995)

[21] M. Tamburini, F. Pegoraro, A. Di Piazza, C. H. Keitel, T. V. Liseykina, and A. Macchi, Nucl. Instrum. Methods Phys. Res., Sect. A 653, 181 (2011).

[22] J. G. Kirk, A. R. Bell, and I. Arka, Plasma Phys. Controlled Fusion 51, 085008 (2009).

[23] L. D. Landau and E. M. Lifshitz, The Classical Theory of Fields (Elsevier, Oxford, 1975).

[24] S. S. Bulanov, T. Z. Esirkepov, A. G. R. Thomas, J. K. Koga, and S. V. Bulanov, Phys. Rev. Lett. 105, 220407 (2010).

[25] S. V. Bulanov, T. Zh. Esirkepov, J. Koga, and T. Tajima, Plasma Phys. Rep. 30, 196 (2004).

[26] I. Gonoskov, A. Aiello, S. Heugel, and G. Leuchs, Phys. Rev. A 86, 053836 (2012).

[27] I. M. Bassett, Opt. Acta 33, 279 (1986).

[28] V. Yanovsky et al., Opt. Express 16, 2109 (2008).

[29] A. Gonoskov, I. Gonoskov, C. Harvey, A. Ilderton, A. Kim, M. Marklund, G. Mourou, and A. M. Sergeev, Phys. Rev. Lett. 111, 060404 (2013).

[30] See Supplemental Material at http://link.aps.org/ supplemental/10.1103/PhysRevLett.113.014801, which includes Refs. [31,32], it contains analytical expressions for the fields of the $e$-dipole pulse, a description of how to approximate such fields in the lab using a finite number of laser beams, and the details of a video demonstrating electrons dynamics in an $e$-dipole pulse.

[31] G. Mourou, B. Brocklesby, T. Tajima, and J. Limpert, Nat. Photonics 7, 258 (2013).

[32] NIF, https://lasers.llnl.gov/. 